\section{Quality of life of older Chilean people living in metropolitan Santiago, Chile: influence of socioeconomic status}

\author{
Daniel Bunout,1 Paulina Osorio,2 \\ Gladys Barrera,1 María José Torrejón,2 \\ Cynthia Meersohn,2 María Sol Anigstein,2 \\ Juan Pablo Miranda, 3 Iris Espinoza, 4 \\ Sandra Hirsch, ${ }^{1}$ María Pía de la Maza,1 \\ 1Institute of Nutrition and Food \\ Technology (INTA); 2Faculty of Social \\ Sciences; 3Faculty of Economy and \\ 4Faculty of Dentistry, University of Chile, \\ Santiago, Chile
}

\begin{abstract}
Quality of life (QOL) of older people is becoming an important public health concern and should be evaluated. The aim of this study was to evaluate socioeconomic and individual determinants of QOL of older subjects living in metropolitan Santiago, Chile. We first carried out a qualitative phase with focus groups of older people. According to the conclusions of the focus groups, a questionnaire about QOL was devised and added to the WhoQoL (World Health Organization Quality of Life) and WHOQoL-Old brief questionnarires. The final document with 85 questions was applied to healthy older subjects living in the community. A total of 1,676 subjects aged $71.8 \pm 7.4$ years (1,189 women) took part in the survey. A multiple stepwise regression model showed that a higher socioeconomic level, a better educational level, performing voluntary work, having a partner, participating in groups with other older people, and being younger were factors independently associated with a higher QOL. A principal components analysis showed that psychological health and social relationships were the main domains that explained the total quality of life score. Psychological health and social relationships were the main determinants of QOL in this sample of older Chilean people living in metropolitan Santiago.
\end{abstract}

\section{Introduction}

In Chile, older people have benefitted from the implementation of sanitary measures, such as public health care programs, immunization campaigns and programs to manage disability, that have been specially designed for them. There is also a public institution committed to the study of older people that aims to solve their specific problems. ${ }^{1}$ As a society's economic and social development reach certain levels and basic sanitary issues are resolved, quality of life (QOL) becomes an issue of concern. QOL is difficult to define and it frequently emphasizes components of happiness and satisfaction with life. ${ }^{2}$ One operational concept defines it as the individual's perception of their position in life in the context of the culture and value systems in which they live, and in relation to their goals, expectations, standards and concerns. ${ }^{3}$

A previous meta-analysis showed that income has a great influence on the subjective wellbeing of older subjects. Other factors that influenced wellbeing were the quality of social relations and having friends. ${ }^{4}$ Studies performed in Chile have shown that older subjects of low socioeconomic status (SES) have a higher frequency of disabilities and cognitive impairment. 5 Therefore, it is important to evaluate the perception of quality of life among subjects with different SES.

Considering that QOL depends on local cultural and environmental factors, it is essential not to extrapolate data from surveys carried out elsewhere and to perform local studies. ${ }^{6}$ The World Health Organization has proposed a standardized methodology to perform local surveys of QOL, but retaining certain common features with other studies, to allow for comparisons. This methodology includes the performance of local focus groups ${ }^{3}$ and adaptation of previously devised questionnaires. ${ }^{7}$

Considering the importance of SES on the conditions of life of older people in Chile, the aim of this study was to evaluate QOL among healthy older Chilean people living independently in metropolitan Santiago, and to explore the socioeconomic and individual determinants and dimensions that affect it.

\section{Materials and Methods}

In the first phase, we created a total of 15 focus groups of 10 subjects each, made up of older Chilean people living in metropolitan Santiago. Five groups met and took part in the survey and these included people of low, medium and high SES. Among participants of each SES level, two focus groups were composed of only men, two with only women and one with both genders. Data from focus groups were analyzed using ATLAS.ti 5.0 qualitative data analysis software (Scientific Software Development, Germany). Participants SES was ascertained according to the classification of the neighborhood where they lived, which is determined by the National Socioeconomic Characterization Survey (Encuesta de Caracterización Socioeconómica Nacional or CASEN) carried out by the Planning Ministry
Correspondence: Daniel Bunout MD, INTA University of Chile, P0 Box 138-11 Santiago, Chile.

Tel. +56.2.978.1485 - Fax: +56.2 .2214030 .

E-mail: dbunout@inta.cl

Key words: aging, quality of life, socioeconomic status, education.

Contributions: DB, PO conception and design of the study, data collection; GB, MJT, CM and MSA data collection; JPB, IE, SH and MPdIM conception and design of the study.

Conflict of interest: the authors declare that they have no competing interests.

Acknowledgements: this work was financed by a grant from Domeyko Program on Health of the University of Chile.

Received for publication: 31 May 2011

Revision received: 8 November 2011.

Accepted for publication: 5 December 2011.

This work is licensed under a Creative Commons Attribution NonCommercial 3.0 License (CC BYNC 3.0).

(C) Copyright D. Bunout et al., 2012

Licensee PAGEPress srl, Italy

Ageing Research 2012; 4:e3

doi:10.4081/ar.2012.e3

of Chile (MIDEPLAN). ${ }^{8}$ The SES characterization of each neighborhood is based on the mean per capita income of its residents. The threshold of poverty is defined as the income required to afford and satisfy predefined basic food requirements. During each focus group meeting, subjects were encouraged to talk about their concept of QOL and the factors that in their opinion influence it.

During the qualitative part of the study, subjects participating in focus groups expressed their appreciation of having a gratifying environment and relationships with friends and family, enjoying good health, being independent, having the resilience to cope with problems, and having good social support provided by the authorities. Based on these opinions, a series of 38 questions about QOL were created. These questions were first tested in a pilot study with 200 older subjects to select suitable categories for the answers. All the questions included in the World Health Organization QOL Questionnaire for older people (WHOQoLOld and WhoQoL BREF) were also included, with the permission of the group that originally devised these questionnaires. ${ }^{9}$ Each question had five possible answers. The complete questionnaire had 85 questions divided into five domains: 8 about family relationships, 18 about environment, 17 about psychological health, 12 about physical health, and 20 about 
social relations. The questionnaire also contained additional questions about the type of income, educational level, number of offspring, type of health insurance, labor status, participation in community centers and voluntary activities. Interviews were carried out in a population based random sample of healthy older subjects aged 60 years old or over, without dementia, defined as a Mini mental score of less than 22,10 and living independently in neighborhoods of high, medium and low income, according to CASEN data. All subjects were interviewed in their homes. Of all the subjects invited to participate, 82 (4.7\%) subjects refused to answer the questionnaire. Subjects living in institutions or nursing homes were excluded from the study. Care was taken to interview the same proportion of men and women in each socioeconomic stratum. The sample size was calculated with an excess number of older people from municipalities of high income, to provide a better contrast with subjects from medium and low income communities. A previous study, in which the Short Form Health Survey (SF-36) had been applied to older Chilean subjects, ${ }^{11}$ required an oversampling of high SES participants in order to obtain differences between groups. This information was used to calculate the sample number. The survey was carried out by trained interviewers who interviewed each subject individually and recorded their answers by filling in a form. Interviews were carried out between March 2009 and March 2010. All the information collected was stored in databases.

For data analysis, each of the answers to a specific question was quantified from 1 to 5 . Five was the highest score given to an answer that corresponded to the subjective perception of better quality of live (i.e. completely satisfied would receive a score of 5 for the question How satisfied are you with your work capacity?). The questions that were not answered were eliminated from the final calculation. Individual scores of answered questions were added up, divided by the maximal score that could be obtained (5) eliminating the unanswered questions and multiplied by 100 . The final score was expressed as a percentage. This calculation was made for each question included in the survey and then separately for each domain (family, environment, psychological health, physical health and social relationships).

The internal consistency of the scale was calculated with Cronbach's alpha, using each individual item of the scale. Internal consistency of the 38 initial questions derived from focus groups and those of the WHOQoLOld and WhoQoL BREF were also calculated.

Differential item functioning analysis ${ }^{12}$ was carried out using a multinomial logistical regression analysis. The categorized score of each domain (classified as good, fair and poor) was included as the dependent variable. Sex,
SES, living alone or with someone else, and age ranges were considered as grouping variables. Total score, also classified as good, fair or poor and was included as the matching variable.

All statistical analyses were performed using Stata 10 for Windows (StataCorp, TX, USA). Variables with a normal distribution are expressed as mean \pm standard deviation; otherwise they are expressed as median (range). Total and individual scores were compared using the Kruskal Wallis test according to sex, educational level, marital and socioeconomic status of participants, living conditions and having versus not having offspring. Those variables that were significantly associated with the score were included in a stepwise multiple regression model in which the total score obtained in the survey was the dependent variable. Socioeconomic, educational and living condition variables that were significantly associated with the score in the univariate analysis were included as independent variables. The threshold for inclusion or rejection of variables in the model was set at $\mathrm{P}=0.05$.

To investigate which of the individual domains had a higher influence in the total score, a principal component analysis using non-standardized data was carried out to determine the main components of the score. The factors obtained were grouped in the same five domains described previously (family, environment, psychological health, physical health and social relationships). The eigenvectors of the first component were used to determine the weight of each domain on the total score, considering that all data were expressed as a percentage of the maximum score, and the variances were very similar.

\section{Results}

A total of 1,676 older subjects took part in the survey. Age, sex and SES of the neighborhood where the participants lived are shown in Table 1. The main income source of the sample was a retirement pension (72\%), 48\% lived with a partner, $20 \%$ lived alone, $91 \%$ had offspring, 45\% had pets, 18\% did voluntary work, and $72 \%$ participated in group activities for older people. Subjects living in neighborhoods of higher SES participated more often in group activities and had a higher educational level $\left(\chi^{2} \mathrm{P}<0.01\right)$. Eight hundred people failed to answer one question and 300 did not answer 2 questions. No participant skipped more than 4 questions. The most frequently unanswered question was about sexual satisfaction.

The alpha value for internal consistency of the whole scale was 0.92 . The values for the 38 initial questions created according to the results of focal groups and for questions from WHOQoLOld and WhoQoL BREF were 0.79,
0.89 and 0.86 , respectively. The median total score of the survey was $69 \%$ (40.6-90.8\%). The figures were $69 \%$ (40.6-90.8\%) among women and $68.8 \%$ (43.9-86.7\%) among men $(\mathrm{P}=\mathrm{NS})$. The values for family domain were $77.5 \%$ (0$100 \%)$, for environmental domain $57.8 \%$ (28.291.1\%), for psychological health domain $71.3 \%$ (34.1-96\%), for physical health domain $76.7 \%$ (26.7-100\%) and for social relationships domain $68 \%$ (31.6-92\%). Differential item functioning analysis did not show any effect of sex, SES, age range and living conditions on the scores of each domain when matching for the total score.

Total score was significantly higher among subjects living in high income neighborhoods, compared with those of medium and low SES, as well as among subjects with a higher educational level, those living with a partner, doing voluntary work and those participating in groups for older people. Having offspring had no influence on the total score (Table 2). A weak but significant negative correlation was observed between age and total score (Spearman's rho=-0.05, P=0.03). A forward stepwise regression model accepted socioeconomic and educational level, doing voluntary work, having a partner, participation in groups of older subjects and age as independent predictors of the total QOL score (Table 3).

Principal component analysis revealed that the scores of psychological health and social relationships had the highest influence on the total QOL score in the total sample and after stratifying by sex, SES and having versus not having a partner (Table 4).

\section{Discussion}

In this survey, QOL of healthy older subjects living independently in metropolitan Santiago was highly influenced by the SES of the neighborhood where the subjects lived. In addition, social relationships and psychological health were the domains with the highest influence on the total QOL score. The overall score obtained in this study is very similar to the score obtained in a Brazilian population using the WHOQoL-OLD 13 and slightly lower than that obtained in European participants when the latter score was developed. ${ }^{6}$ A recent study performed in Colombia also showed a close association between health related QOL and environmental features that are characteristics of high SES neighborhoods, such as park areas and recreation facilities. ${ }^{14}$

This study followed the guidelines of the World Health Organization that recommends the use of focus groups with local participants in order to determine, through a qualitative analysis, which aspects should be included in a Q0L survey.6 This methodology allows certain 
areas that may be important in a determined social setting and that cannot be generalized to be emphasized. ${ }^{3}$ Therefore, we included a series of questions that were important to participants in the focus groups, but retained the original questionnaires elaborated by the World Health Organization. The alpha values of the subscales of the questionnaire were appropriate since the lowest correlation (square root of alpha) was 0.88 . The questionnaire was relatively long and took approximately $30 \mathrm{~min}$. The Cronhnbach alpha of over 0.9 indicates that there was an overlap in some questions, and effectively this was true. This allows certain answers to be confirmed and reduces the impact of opinions which are not true.

We used the SES of the neighborhood where the older subjects lived as a proxy of their own SES. We decided to use this parameter because the actual level of income of the participants may be distorted by the low retirement pensions. The use of income as an indicator of SES would have meant that some participants would have been misclassified, especially former professionals with a high level of education who experienced a drastic drop in their incomes when they retired. The classification of poverty in Chile depends almost entirely on income levels. 5 There was a strong association between QOL score, socioeconomic and educational level of participants. This fact illustrates the inequity that still prevails in Chile. Other authors have previously reported an association between SES, cognitive status, mental health, QOL, self-perceived health and mortality.5,15-16 The independent income ratio between the higher and lower income quintile in Chile has varied from 20 in 1990 to 24 in 2009.8 This is an indication of how the gap between rich and poor is increasing. Probably, the most important implication of this study is that we not only have to improve the economic and social conditions of the elderly, but also to reduce the social inequities in our society.

We used principal component analysis to determine which domain of the survey had a greater influence on the total score. Psychological health and social relationships emerged as the most influential domains. These findings highlight the need to conduct local surveys to assess determinants of QOL, since a recently published survey found contradictory results among older Spanish people. ${ }^{17}$

Social relationships were also confirmed as an important determinant of QOL. These results may be contaminated by the fact that participation in group activities was more common among more affluent populations of older people. However, even considering this source of error, social activities are valued by older people as an important asset in their lives. Older subjects recognize the importance of having friends and there is a negative association between the number of friends and the prevalence of depression. ${ }^{18}$ In Chile, a travel program for older people improved the sense of wellbeing among participants. ${ }^{19}$ These results should encourage the development of more group activities for the elderly that are easy and inexpensive to implement. Considering the importance given by older people to social relationships, efforts should be directed to achieving a better integration of this age group in society, providing opportunities for work, social events and reducing the discrimination against old age.

Table 1. Number, sex and age (expressed as mean \pm standard deviation) of interviewed participants.

\begin{tabular}{lcccc}
$\begin{array}{l}\text { Socioeconomic level } \\
\text { of the neighborhood } \\
\text { where the subject lives }\end{array}$ & N. participants & Age & N. participants & Age \\
High & 630 & $73 \pm 7$ & 248 & $72 \pm 7$ \\
Medium & 358 & $72 \pm 8$ & 156 & $70 \pm 7$ \\
\hline Low & 201 & $71 \pm 8$ & 83 & $69 \pm 7$ \\
\hline
\end{tabular}

Table 2. Quality of life scores (expressed as median and range of percentages of maximum possible score) in studied participants according to socioeconomic, educational and family features.

\begin{tabular}{lccc} 
Variable & Categories (n) & Q0L score & $\begin{array}{c}\text { P between } \\
\text { categories }\end{array}$ \\
Socioeconomic level & High (878) & $70.8 \%(40.6-90.8 \%)$ & $<0.01$ \\
& Medium (514) & $66.9 \%(44.8-86.8 \%)$ & \\
Educational level & Low (n=284) & $65.6 \%(48-81.9 \%)$ & \\
& Illiterate (29) & $65.1 \%(47.4-77.2 \%)$ & $<0.01$ \\
& Basic (466) & $66.3 \%(40.6-85.9 \%)$ & NS \\
& High school (880) & $69.5 \%(43.9-90.8 \%)$ & \\
Has offspring & University (292) & $71.9 \%(50-89.7 \%)$ & $<0.01$ \\
& Yes (1527) & $69 \%(40.6-89.7 \%)$ & $<0.01$ \\
Does voluntary work & No (149) & $67 \%(40.6-86.7 \%)$ & \\
& Yes (297) & $71 \%(47.5-89.7 \%)$ & \\
\hline Living status & No (1379) & $68.5(40.6-90.8 \%)$ & $<0.01$ \\
& Living alone (337) & $69.3(44.8-90.8 \%)$ & \\
Participates in groups & Living with a partner (801) & $69.7(43.9-89.7)$ & \\
for older people & Living with relatives (538) & $67.6(40.6-86.7)$ & \\
\hline
\end{tabular}

Table 3. Stepwise multiple regression model of variables independently influencing the total quality of life scores of participants.

\begin{tabular}{|c|c|c|c|c|c|c|}
\hline $\begin{array}{l}\text { Variables accepted } \\
\text { in the model }\end{array}$ & Coefficient & $\begin{array}{l}\text { Standard } \\
\text { error }\end{array}$ & $\mathrm{t}$ & $\mathbf{P}$ & \multicolumn{2}{|c|}{$\begin{array}{l}95 \% \text { Confidence } \\
\text { intervals }\end{array}$} \\
\hline $\begin{array}{l}\text { Socioeconomic level of the } \\
\text { neighborhood where the subject lives }\end{array}$ & 2.21 & 0.23 & 9.59 & $<0.01$ & 1.76 & 2.66 \\
\hline Educational level & 1.01 & 0.17 & 5.91 & $<0.01$ & 0.67 & 1.34 \\
\hline Doing voluntary work & 1.93 & 0.43 & 4.47 & $<0.01$ & 1.08 & 2.77 \\
\hline Having a partner & 1.58 & 0.33 & 4.82 & $<0.01$ & 0.94 & 2.22 \\
\hline Participation in groups of older people & 1.52 & 0.37 & 4.12 & $<0.01$ & 0.79 & 2.24 \\
\hline Age & -0.05 & 0.02 & -2.22 & 0.03 & -0.09 & -0.01 \\
\hline Constant & 61.62 & 1.74 & 35.36 & $<0.01$ & 58.20 & 65.04 \\
\hline $\mathrm{R}^{2}$ of the model & $0.14(\mathrm{P}<0.01)$ & & & & & \\
\hline
\end{tabular}

The low value attributed to the family comto other domains is worthy of note. This tions and experiences of independent older people in Chile, since they had tended to rely offspring for social, economic and emotionsons and cou the care of their parents, due to burdens ofed by their own work and offspring, is probably forcing older people to become more independent and self-confident. This would 
Table 4. Principal component analysis of the influence of specific domains on the total QOL scores. Eigenvectors of the first component for each domain are shown. Bold numbers represent the two highest values for each category.

\begin{tabular}{lcccccc} 
& & Family & $\begin{array}{c}\text { Environment } \\
\text { health }\end{array}$ & $\begin{array}{c}\text { Psychological } \\
\text { health }\end{array}$ & $\begin{array}{c}\text { Physical } \\
\text { relationships }\end{array}$ & Social \\
\multicolumn{2}{l}{$\begin{array}{l}\text { Total sample } \\
\text { Gender }\end{array}$} & 0.34 & 0.38 & 0.52 & 0.46 & 0.50 \\
\multicolumn{2}{l}{ Men } & 0.35 & 0.38 & 0.52 & 0.46 & 0.50 \\
& Women & 0.33 & 0.38 & 0.52 & 0.46 & 0.50 \\
\hline
\end{tabular}

Socioeconomic level

of the neighborhood

where the subject lives

\begin{tabular}{rlllll} 
High & 0.35 & 0.37 & 0.52 & 0.46 & 0.51 \\
Medium & 0.31 & 0.38 & 0.53 & 0.47 & 0.51 \\
Low & 0.34 & 0.35 & 0.55 & 0.46 & 0.50 \\
Lives with partner & & & & & \\
Yes & 0.32 & 0.40 & 0.52 & 0.46 & 0.50 \\
No & 0.35 & 0.36 & 0.52 & 0.46 & 0.51 \\
\hline
\end{tabular}

reinforce the relevance of social relations for the QOL of older people. In fact, a study performed in Unites States showed that only $12 \%$ of a large sample of subjects had some sort of family caregiving responsibility. 20 We can understand why people avoid taking care of elderly family members considering the detrimental effect on Q0L that this has on caregivers. ${ }^{21,22}$

A limitation of these results is the low $R$ square obtained in the multiple regression model. Probably in the future more environmental variables, such as those reported in the Colombian study, 14 and more information about income levels could improve the predictive capacity of the model.

In conclusion, QOL of older Chilean people living in metropolitan Santiago is highly dependent on SES and educational level, and is fundamentally related to psychological wellbeing and maintaining social relationships. These results should help to direct the efforts of authorities interested in improving the wellbeing of this age group living in a metropolitan city.

\section{References}

1. Senama. 2010. Available from: http://www. senama.cl.

2. Fayers P, Machin D. Quality of Life: The Assessment, Analysis and Interpretation of Patient-reported Outcomes, 2nd ed. John Wiley and Sons, NJ, USA, 2007.

3. WHOQOL-BREF. Introduction, admini-stration, scoring and generic versions of the assessment. Field trial version. World Health Organization, Geneve, 1996.

4. Pinquart M, Sörensen S. Influences of socioeconomic status, social network, and competence on subjective well-being in later life: a meta-analysis. Psychol Aging 2000;15: 187-224.

5. Albala C, Sanchez H, Lera L, et al. Efecto sobre la salud de las desigualdades socioeconómicas en el adulto mayor. Resultados basales del estudio expectativa de vida saludable y discapacidad relacionada con la obesidad (Alexandros). Rev Med Chile. In press.

6. Power M, Quinn K, Schmidt S; WHOQOLOLD Group. Development of the WHOQOLold module. Qual Life Res 2005;14:2197214.

7. WHOQOL user manual. Available from: http://depts.washington.edu/yqol/docs/WH 0Q0L Bibliography.pdf

8. Encuesta de Caracterización Socioeconómica Nacional. Documento Metodológico. Ministerio de Planificación Nacional. Gobierno de Chile. Available from: http://www.mideplan.cl/casen/pdf/metodologia_2006.pdf

9. Hawthorne G, Davidson N, Quinn K, et al. Issues in conducting cross-cultural research: implementation of an agreed international protocol [corrected] designed by the WHOQOL Group for the conduct of focus groups eliciting the quality of life of older adults. Qual Life Res 2006;15:125770.

10. Folstein MF, Folstein SE, McHugh PR. "Mini mental state": a practical method for grading the cognitive state of patients for the clinician. J Psychiatr Res 1975;12:18998.

11. Albala C, Garcia C, Lera L. Encuesta sobre Salud, Bienestar y Envejecimiento en Santiago de Chile (Estudio SABE en Chile) (Survey on health, wellbeing and aging in Santiago, Chile (SABE study in Chile)). Santiago, OPS/INTA/U, de Chile, 2007.
12. Scott NW, Fayers PM, Aaronson NK, et al; EORTC Quality of Life Group and the Quality of Life Cross-Cultural MetaAnalysis Group. Differential item functioning (DIF) analyses of health-related quality of life instruments using logistic regression. Health Qual Life Outcomes 2010;8:81.

13. Margis R, Donis KC, Schönwald SV, Rieder CR. WHOQOL-OLD assessment of quality of life in elderly patients with Parkinson's disease: influence of sleep and depressive symptoms. Rev Bras Psiquiatr 2010;32:12531.

14. Parra DC, Gomez LF, Sarmiento OL, et al. Perceived and objective neighborhood environment attributes and health related quality of life among the elderly in Bogotá, Colombia. Soc Sci Med 2010;70:1070-6.

15. Subramanian V, Delgado I, Jadue L et al. Income inequality and self rated health: an analysis from a contextual perspective in Chile. Rev Med Chil 2003;131:321-30.

16. Mardones F. Inequality of health care for the elderly in Chile. Rev Med Chil 2004; 132:865-72.

17. Jadresic E, Araya R. Prevalence of postpartum depression and associated factors in Santiago, Chile. Rev Med Chil 1995;123: 694-9.

18. Leiva MJ, Fuentealba C, Boggiano C, et al. Quality of life of patients subjected to gastric bypass more than one year ago: influence of socioeconomic status. Rev Med Chil 2009;137:625-33.

19. Elosua P. Subjective values of quality-oflife dimensions in the elderly. Rev Esp Geriatr Gerontol 2010;45:67-71.

20. Demura $S$, Sato $S$. Relationships between depression, lifestyle and quality of life in the community dwelling elderly: a comparison between gender and age groups. J Physiol Anthropol Appl Human Sci 2003;22:159-66.

21. Marín PP, Carrasco M, Cabezas M, et al. [Biomedical impact of traveling for Chilean elderly. Rev Med Chil 2004;132: 573-8.

22. Roth DL, Perkins M, Wadley VG, et al. Family caregiving and emotional strain: associations with quality of life in a large national sample of middle-aged and older adults. Qual Life Res 2009;18:679-88.

23. Arai H, Nagatsuka M, Hirai K. The relationship between health-related quality of life and social networks among Japanese family caregivers for people with disabilities. Biopsychosoc Med 2008;2:17.

24. Schulz R, Mendelsohn AB, Haley WE, et al. Resources for Enhancing Alzheimer's Caregiver Health Investigators. End-of-life care and the effects of bereavement on family caregivers of persons with dementia. N Engl J Med 2003;349:1936-42. 